\title{
Cardiomyopathy in farmed Norwegian salmon
}

\author{
H. W. Ferguson ${ }^{1}$, T. Poppe ${ }^{2}$, D. J. Speare ${ }^{1}$ \\ ${ }^{1}$ Department of Pathology, Ontario Veterinary College, University of Guelph, Ontario, Canada N1G 2 W1 \\ ${ }^{2}$ National Veterinary Institute, Box 8156 Dep, N-0033 Oslo 1, Norway
}

\begin{abstract}
This paper describes the gross findings, histopathology, and ultrastructural and scanning electron microscopical (SEM) appearance of farmed Norwegian Atlantic salmon dying from a disease known locally as 'acute heart failure' Pathological findings were mainly cardiac, and some fish showed haemopericardium due to rupture of the atrial wall. Lesions were largely restricted to the spongy portion of ventricle and atrium, and comprised myocardial degeneration and necrosis, with variable degrees of endocardial-associated hypercellularity, plus macrophage and lymphocyte infiltration; epicarditis was also present in most fish. Transmission electron microscope findings confirmed the myofibrillar disruption, while the SEM showed crater-like discontinuities in the endocardial plasmalemma. The cause and pathogenesis of these remarkably severe lesions are unknown; whether they are primarily degenerative or inflammatory must await a more in-depth study. Until more is known, use of the term cardiomyopathy syndrome would seem most appropriate.
\end{abstract}

\section{INTRODUCTION}

During the last 5 to $6 \mathrm{yr}$, increasing numbers of Norwegian fish farms (100 so far) have been affected by a disease known locally as 'acute heart failure in salmon' Typically, the condition occurs in the autumn months, affecting fat and fast-growing Atlantic salmon Salmo salar in sea-cages, usually 14 to $18 \mathrm{mo}$ after their introduction to seawater. Compared to other conditions, the fish frequently die with little reported evidence of prior clinical disease. The present paper describes the gross findings and histopathology in one such outbreak of disease, as well as the transmission and scanning electron microscopical (TEM and SEM) appearance of the cardiac lesions.

\section{MATERIALS AND METHODS}

Necropsies were conducted in October on 12 moribund fish and 4 clinically healthy ones from a single cage site from which increased mortality had been reported. Since their introduction to seawater 18 mo previously, the fish had been fed a commercially prepared pelleted diet. Tissues for light microscopical examination were fixed at $4{ }^{\circ} \mathrm{C}$ in $2.5 \%$ glutaraldehyde in a phosphate buffer ( $\mathrm{pH} 7.4$ and total osmolality ca $320 \mathrm{mOsm}$ ). Organs sampled included heart, liver, pyloric caeca and pancreas, kidney, spleen, and lateral line musculature with both red and white muscle. Tissues were subdivided and subsequently processed in paraffin wax for routine sectioning and staining with haematoxylin and eosin ( $\mathrm{H} \& \mathrm{E}$ ) or Masson's trichrome. Alternatively they were embedded in methacrylate resin and $1 \mu \mathrm{m}$ sections stained with $\mathrm{H} \& \mathrm{E}$, or they were post-fixed in $1 \%$ osmium tetroxide for examination in either TEM or SEM. Those samples destined for the SEM were dehydrated in graded ethanols, criticalpoint dried and sputter coated with gold palladium, while those for the TEM were dehydrated in graded ethanols and embedded in Epon resin. Ultrathin sections were stained with uranyl acetate and lead citrate.

\section{RESULTS}

\section{Moribund fish}

External gross lesions were usually lacking. On internal examination, a fibrinous perihepatitis and an enlarged or ruptured atrium with consequent haemopericardium were the only lesions found (Fig. 1). Histopathological examination, however, revealed significant changes, and these were mainly restricted to the spongy myocardium of both atrium and ventricle, leaving intact the ventricular compact layer. Lesions varied in severity and distribution from mild and multifocal to massive and severe, affecting all spongy trabeculae in both atrium and ventricle. Individual 
hearts, however, often had different stages of reaction in different areas or trabeculae.

Mild lesions were characterized by multifocal granular degeneration of fibres, with loss of striation. This change was accompanied by the formation of an amorphous coagulum which was hyper-eosinophilic in paraffin sections but pale in $\mathrm{H} \& \mathrm{E}$ stained methacrylate sections

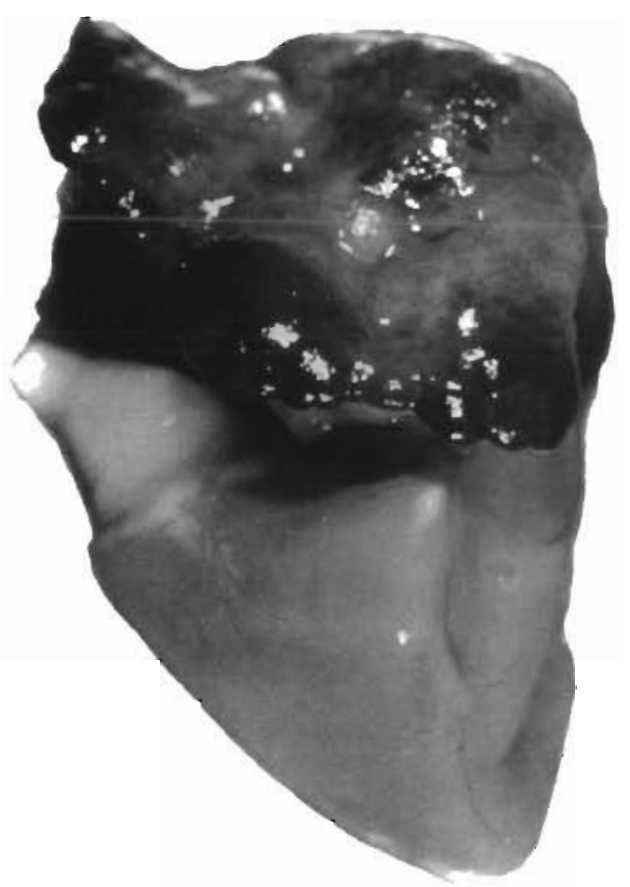

Fig. 1. Salmo salar. Heart from fish with cordiomyopathy syndrome showing massive thrombosis of atrium
(Fig 2). Vacuolation was also present, especially of the sub-endocardial space. Hypercellularity of these degeneratc and necrotic foci was mainly due to the presence of small rounded cells with hyperchromatic nuclei, probably lymphocytes, macrophages, and possibly also subendocardial cells. In the less severely affected fish these were located mostly in the sub-endocardial space, but in severe cases they were also present within the fibres; in necrotic fibres which were comprised of little more than an endothelial-delimited tube of debris, macrophages were seen to contain eosinophilic granular material, or yellow-brown ceroid or lipofuscin-like pigment, probably the remnants of phagocytosed necrotic myocytes (Fig. 3). Lysis of contractile fibres did not seem to be immediately accompanied by nuclear loss; some pyknosis and karyorrhexis were indeed present, but whethet of myocytes or not was unclear. In some fish there was tremendous prominence of endocardium and subendocardium, and individual myocytes often had massively hypertrophied nuclei and nucleoli (Fig. 4). This latter change, which was thought to represent compensatory hypertrophy, was sometimes accompanied by trabecular fibrosis, again possibly and attempt at repair (Fig, 5). Overall mitotic activity was low, and where seen, was associated with the endocardium.

In severely affected hearts, the extent of the necrosis and associated response was truly impressive; atrial thrombosis was common in these cases, and occasionally it was extensive enough to obliterate much of this compartment. In many hearts, necrosis of myocardium was accompanied by marked trabecular hypercellularity, but in others, this was much less pronounced, and it was felt that this may possibly represent an early stage of the disease process.

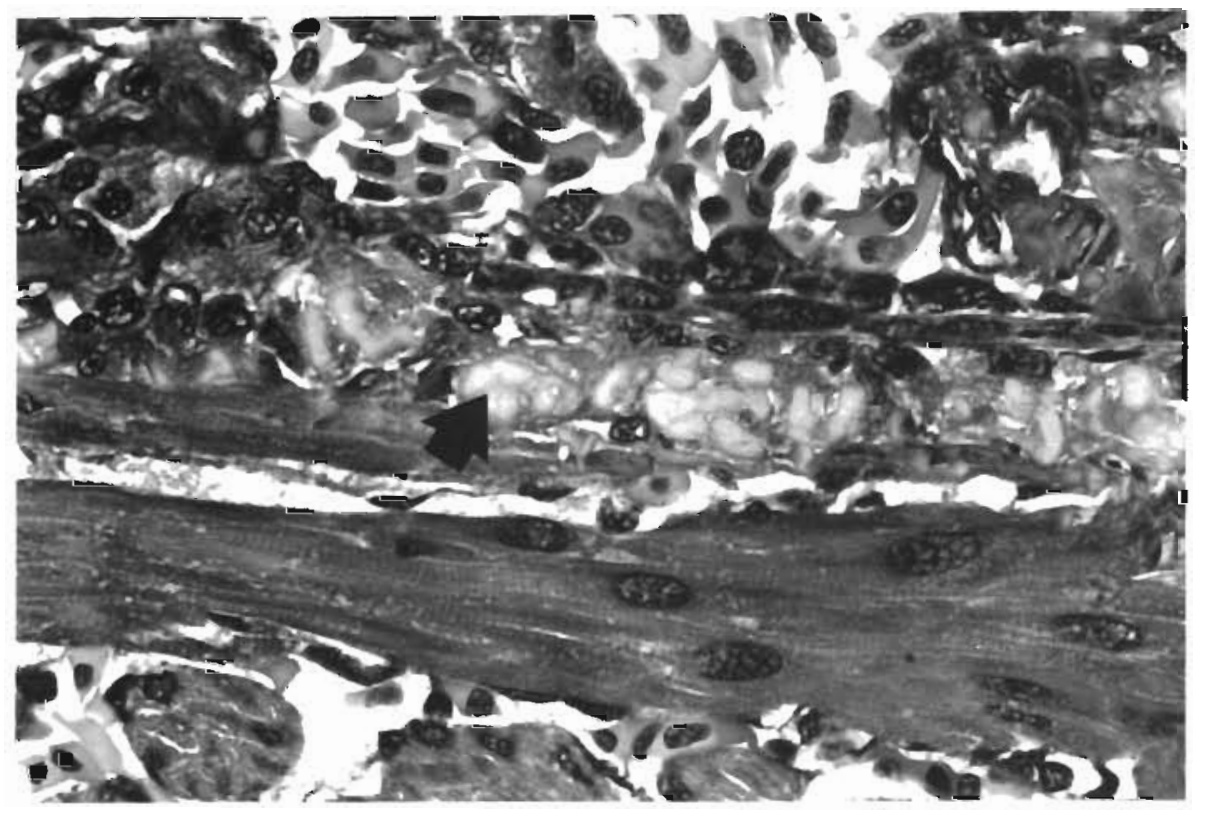

Fig. 2. Salmo salar Methacrylate section of ventricle from fish with early lesions showing acute lysis of myocardium (arrow). H \& $E \times 450$ 


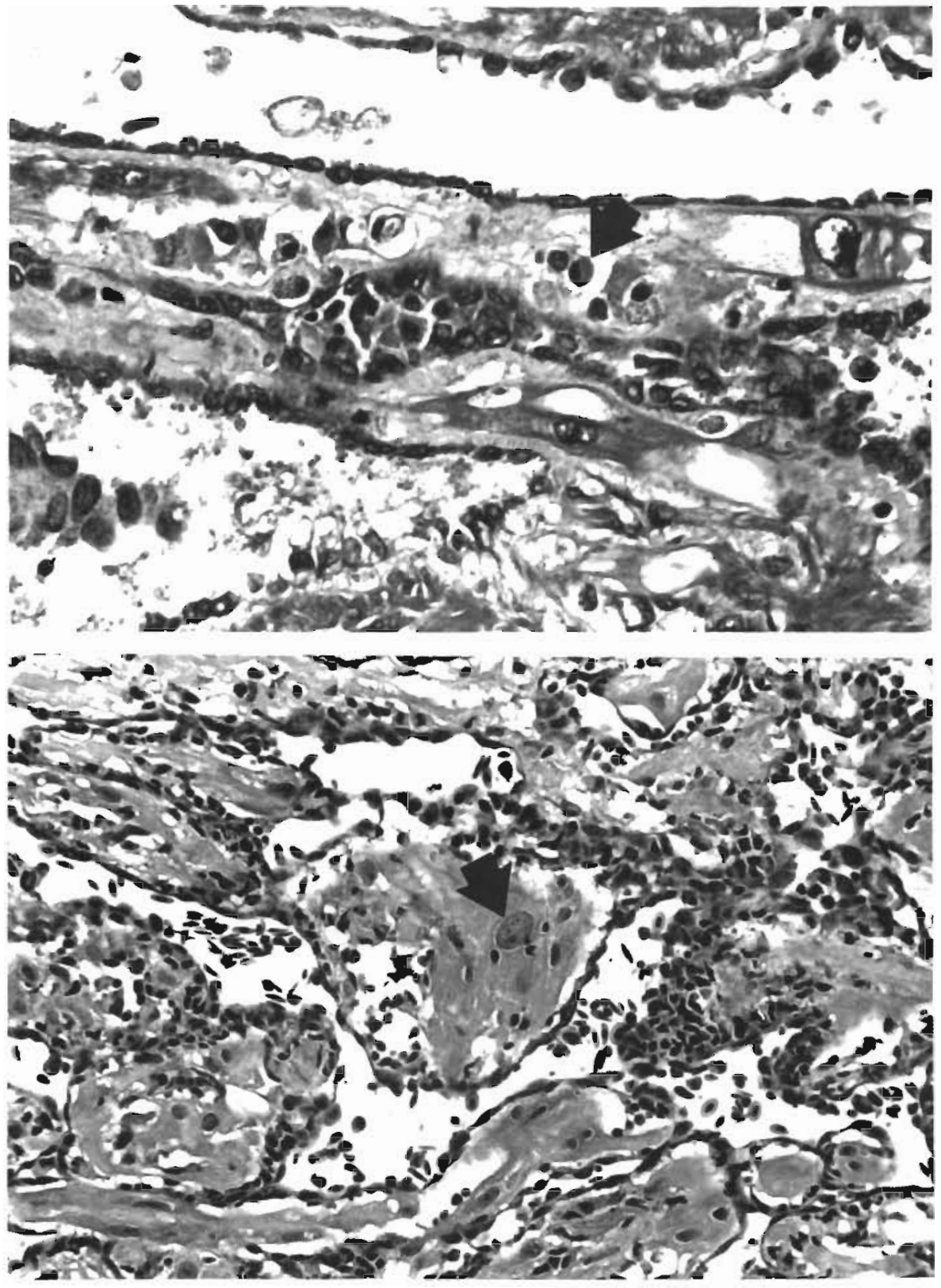

Fig. 3. Salmo salar. Ventricle from severely affected fish showing loss of myofibres and the presence of large numbers of mononuclear cells (macrophages?) containing ceroid-like pigment (arrow). H\&E $\times 450$
Other features of the disease included hypercellularity beneath the epicardium, and in some fish this was more prominent than the myocardial lesions. The reaction was composed of macrophages, lymphocytes and possibly plasma cells, eosinophilic granular cells, and a few neutrophils. The response was usually diffuse, but sometimes it extended into the compact myocardium or formed discrete foci, often associated with the coronary vessels, which in some cases had necrotic walls. In some fish there was a more diffuse scattering of small mononuclear cells throughout the compact layer, but for the most part the myocardium remained normal.

Other lesions were present in the kidney and liver of most fish; these latter were consistent with those expected with a failing circulation. In the liver, there was periacinar degeneration and necrosis; a mild fibrinous coat was sometimes present on the capsule. Lesions in red or white skeletal muscle were notably limited, as were any major changes in the pancreas although small clusters of neutrophils were sometimes found within blood vessels.

Little further insight into the development or pathogenesis of these lesions was gained in TEM, except to confirm the lysis of the myofibrils (Fig. 6). In SEM however, the plasmalemma of the apparently swollen endothelial cells had numerous pseudopod-like fingers 

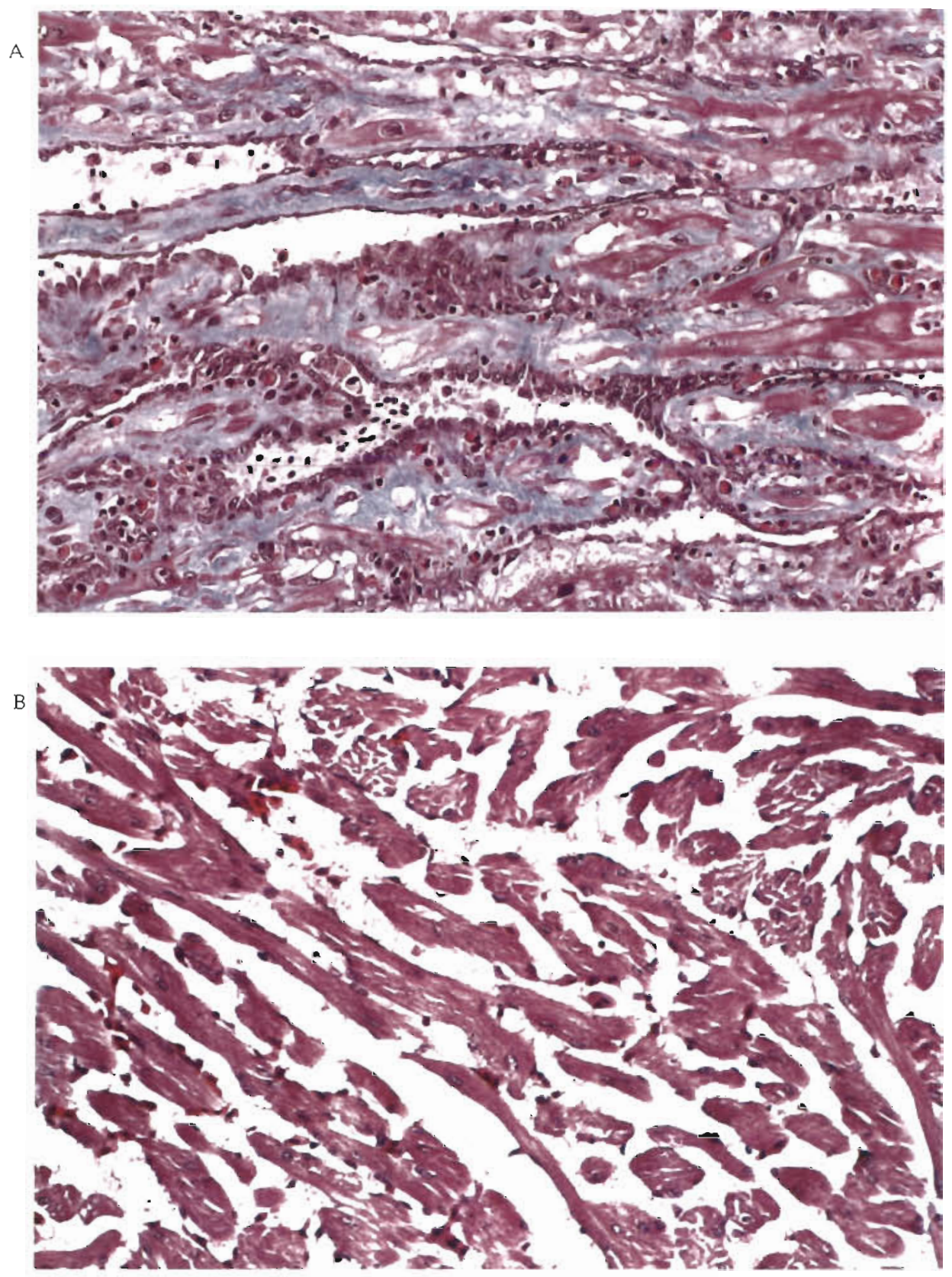

Fig. 5. Salmo salar. (A) Ventricle from fish with pronounced fibrosis (blue); numerous debris-laden macrophages may also be seen, many situated beneath the prominent endocardium. (B) Normal tarmed fish of the same age. Masson's trichrome

and ruffles; in addition however, crater-like discontinuities were seen on the surface of many cells (Fig. 7)

\section{Clinically normal fish}

There were no external lesions, but histologically it was determined that all 4 had evidence of mild to moderate cardiomyopathy similar to that described for moribund fish. No other lesions were seen in these fish.

\section{DISCUSSION}

There seems little reason to doubt that the cause of mortality in these fish can be attributed to the cardiac lesions. Severe thrombosis by itself would cause heart failure, but the extent of the necrosis was such that in many cases, perforation of the atrial wall with haemopericardium and cardiac tamponade were almost inevitable sequelae, especially in a chamber with no compact layer. 


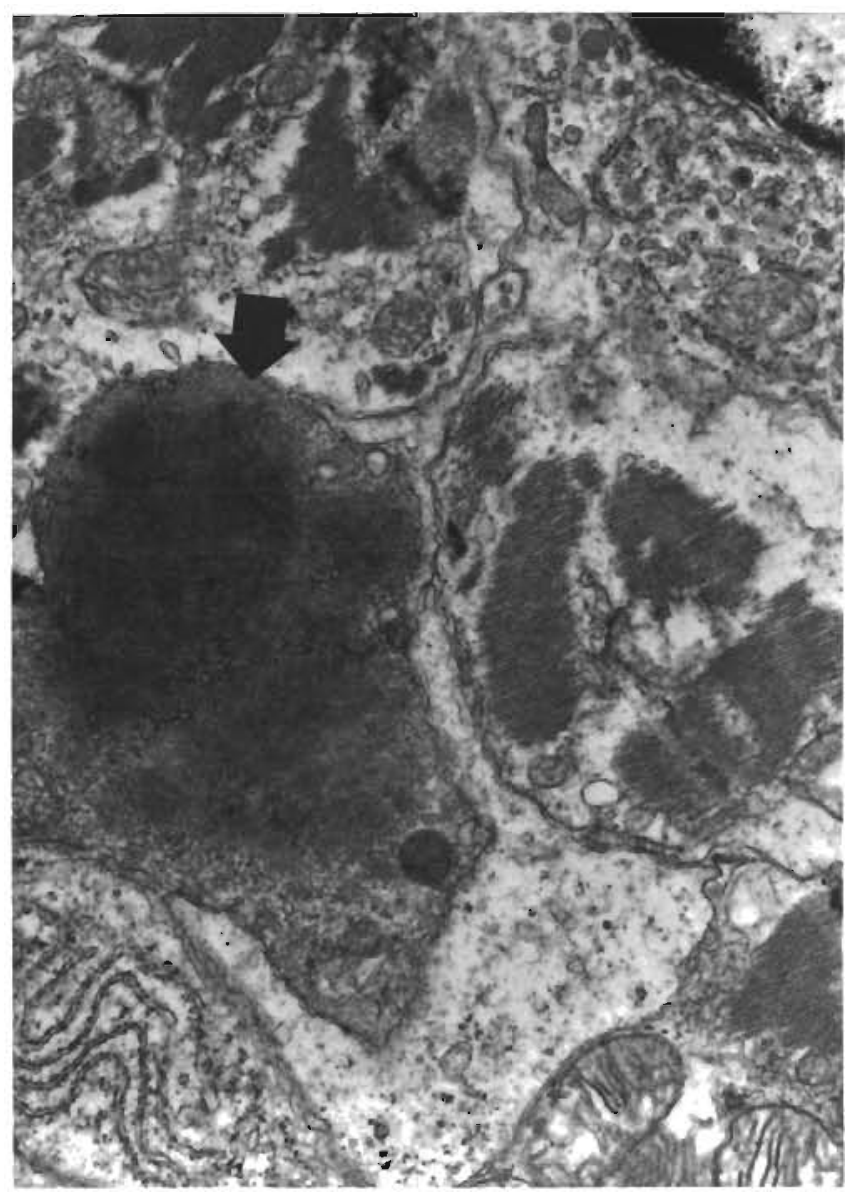

Fig. 6. Salmo salar. Ventricle from fish with cardiomyopathy syndrome showing lysis of myofibrils (arrow); for comparison an adjacent fibre remains relatively normal. $\times 10000$
This is a uniquely severe cardiac disease. Accordingly, it makes interpretation very difficult, as we have very little to compare it with in any vertebrate. The problem is compounded by the fact that we have almost no idea of the time course involved. From a strictly morphological viewpoint, most fish had a severe degenerative and necrotizing myocardial disease in which inflammation, largely chronic in nature, also played a major role, although this did vary from fish to fish. To merely view it in these terms, however, begs the fundamental questions about the pathogenesis. Firstly, is it primarily a degenerative condition of cardiac myocytes with resulting inflammation, or is it the converse? Secondly, is the endocardium the major target or is it the myocardium? The answers to both of these questions are purely speculative at this time.

Despite the fact that myocardial degeneration with ongoing necrosis will, in mammals, sometimes elicit a moderate inflammation (Robinson \& Maxie 1985), the extent of the trabecular (interstitial) and epicardial hypercellularity in many of these fish favours the idea of a primary myocarditis and an accompanying epicarditis. On the other hand, some fish had advanced myolysis with minimal hypercellularity; could this be an early lesion? Moreover, the identity of many of the involved cells remains to be determined, and while lymphocytes and macrophages were certainly present, the latter removing necrotic debris from within the spongy trabeculae, many of the smaller mononuclear cells were of uncertain lineage: the potential for growth in the teleost heart is only just starting to be understood (Farrell et al. 1988) and the possibility that many of the cells were reserve or
Fig. 7. Salmo salar. Atrium from fish with cardiomyopathy syndrome showing crater-like discontinuities and erosions of the endocardium (arrow) the surface of which shows numerous pseudopodia and ruffles

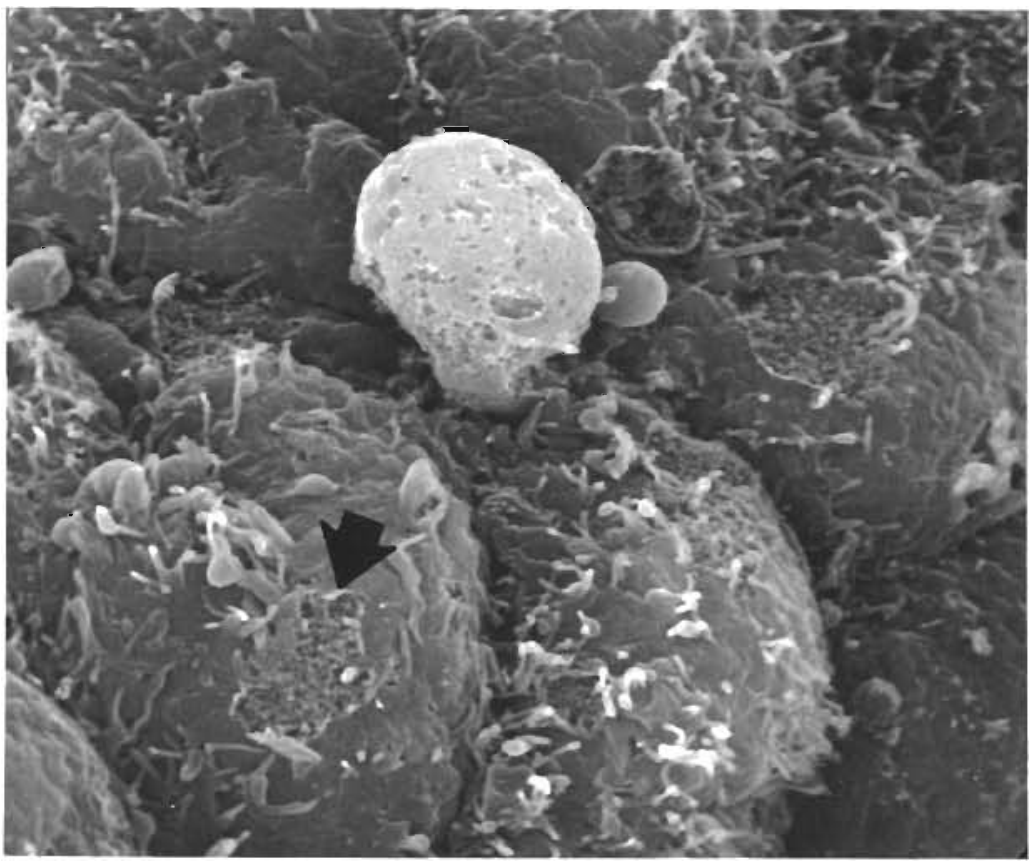


satellite cells attempting repair or regeneration must be considered. Similarly, the heart can be recruited for haemopoiesis in times of high demand, and separating stem cells of different lineages is a difficult task. Although these were well-grown fish, it must also be appreciated that the relative proportions of spongy to compact ventricular myocardium normally change as Atlantic salmon grow (with a decline in spongy) and that this may have an influence, possibly a profound one, on the course of events (Poupa et al. 1974).

Regardless of whether this is primarily an inflammatory or a degenerative condition, is the myocardium merely becoming involved as an innocent bystander in a reaction directed at or orchestrated by the endocardium? The pronounced cellularity immediately adjacent to the endocardium, at times forming almost a cuff around the enclosed myofibres, certainly suggests a marked degree of reactivity in either the endocardium itself or the subendocardial cells. Taken together with the SEM evidence of 'cratering' in the endocardium, and the fact that the compact myocardium remained relatively unscathed regardless of the severity of the reaction elsewhere, this tends to point to a primary endocardial disease. On the other hand, it must also be remembered that spongy myocardium is metabolically very different from compact (Ewart \& Driedzic 1987) not least because of its quite distinct blood supply (arterial blood to compact, and venous to spongy), and that it could therefore be expected to respond differently. In this connection it is worth noting that fish heart has significant levels of cytochrome p450, second only to the liver in those species examined (Stegeman et al. 1982) and that most of this activity probably resides in the endocardium. These enzymes are of course important in the biotransformation of exogenous compounds as well as endogenous compounds such as steroids. Possibly of more importance, however, from an antigen trapping or presenting standpoint, is the fact that the teleost endocardium is phagocytic, although this is less pronounced in the ventricle than in the atrium (Ferguson 1975) and is more obvious in teleosts such as marine flatfish, than it is in salmonids. While the large surface area of the tissue facilitates both biotransformation and antigen trapping, at the same time it renders the myocardium vulnerable should the endocardium become damaged.

No evidence was found, at either the light or electron microscopic level, for the presence of any infectious agent; by comparison with similar diseases in mammals, however, the changes are not inconsistent with an infectious etiology, nor indeed with an immune-mediated, toxic, nutritional, catecholamine, or possibly anoxic one; at this stage it would seem that almost nothing can be ruled out. As a consequence, the name cardiomyopathy syndrome (CMS)' would seem most apt.
How does this disease compare to 'pancreas disease' (PD) of Salmo salar, another condition of intensively reared Atlantic salmon (Munro et al. 1984, McVicar 1987 ) in which the heart (and other striated muscles) may also be involved, and which can sometimes be found with CMS on the same facilities? Apart from the facts that in the present case neither the pancreas nor skeletal muscle was inyolved to any significant degree, and that this disease affected fat and well-grown fish (whereas PD mainly targets fish in their first year in salt water), the major apparent difference lies in the severity and extent of the cardiac lesions; in one case of PD (Ferguson et al. 1986b) the hypercellularity and associated myocardial degeneration and coagulative necrosis were relatively subdued compared to most (but by no means all fish in the present case. Moreover, in a review of the lesions associated with $\mathrm{PD}$ on a large number of farms, McVicar (1987) reported that cardiomypopathy was an inconstant finding. Another difference from PD may lie in the blood chemistry; preliminary results show that fish with CMS have normal plasma vitamin $E$ levels compared to dramatically low levels in Norwegian fish with PD. These latter are similar therefore to the low levels which accompanied a single outbreak of PD in Scotland (Ferguson et al. 1986a) and which showed that low circulating vitamin E levels can be associated with the syndrome, although whether as a result of the disease (due to consumption) rather than as a cause (due for example to impaired uptake) remains unclear. The presence of pancreatic lesions in PD may help to explain some of the differences from CMS in clinical chemistry, pancreatic enzymes being necessary for proper utilisation of vitamin E esters, as these are the common form of supplementation in commercially prepared salmonid diets (Hung et al. 1982). Thus, in summary, these 2 syndromes seem to have quite distinct clinical and pathological presentations, but the possiblity nevertheless remains that they merely represent extreme ends of the spectrum of the same disease, with the same but as yet undetermined aetiology. At the present time, PD is considered (McVicar 1987) to be distinct from infectious pancreatic necrosis (PN), caused by a birnavirus. It should be pointed out however, that in Norway, high titres to this virus are found in fish with PD as well as in those with CMS (Poppe et al. 1989). The significance of this finding has yet to be determined, as IPN virus is very widespread in Norwegian aquaculture and associated disease regarded as negligible (Krogsrud et al. 1988).

This report has described the lesions seen in a relatively small number of fish from a single site. Much more work needs to be done to extend these observations to other outbreaks of CMS at other sites, to examine the time-course involved, and to compare and 
contrast this syndrome with PD from a biochemical and epidemiological perspective, as well as a pathological one.

Acknowledgements. We thank Dr Colin Cowey of the Nutrition Department, University of Guelph for helpful advice concerning aspects of vitamin E metabolism. The Fish Pathology Laboratory receives much of its funding from the Ontario Ministry of Agriculture and Food.

\section{LITERATURE CITED}

Ewart, H. S., Driedzic, W. R. (1987). Enzymes of energy metabolism in salmonid hearts: spongy versus cortical myocardia. Can. J. Zool. 65: 623-627

Farrell, A. P., Hammons, A. M., Graham, M. S., Tibbits, G. F. (1988). Cardiac growth in rainbow trout, Salmo gairdneri. Can. J. Zool. 66: 2368-2373

Ferguson, H. W. (1975). Phagocytosis by the endocardial lining cells of the atrium of plaice (Pleuronectes platessa). J. comp. Pathol. 85: 561-569

Ferguson H. W., Rice, D. A., Linas, J. K. (1986a). Clinical pathology of myodegeneration ('pancreas disease') in Atlantic salmon (Salmo salar). Vet. Rec. 119; 297-299

Ferguson, H. W., Roberts, R. J., Richards, R. H., Collins, R. O., Rice, D. A. (1986b). Severe degenerative cardiomyopathy associated with pancreas disease in Atlantic salmon, Salmo salar L. J. Fish Dis. 20: 95-98

Responsible Subject Editor: Professor O. Kinne, Oldendorf Luhe, F. R. Germany
Hung, S. S. O., Moon, T W., Hilton, J. W., Slinger, S. J. (1982). Uptake, transport and distribution of DL- $\alpha$-tocopheryl acetate compared to $\mathrm{D}$ - $\alpha$-tocopherol in rainbow trout (Salmo gairdneri). J. Nutr. 112: 1590-1599

Krogsrud, J., Hastein, T., Ronningen, K. (1988). Infectious pancreatic necrosis virus in Norwegian fish farms. Abstracts of the International symposium on viruses of lower vertebrates. Munich

McVicar, A. E. (1987). Pancreas disease of farmed Atlantic salmon, Salmo salar, in Scotland: epidemiology and early pathology. Aquaculture, Amsterdam 67: 71-78

Munro, A. L. S., Ellis, A. E., McVicar, A. E., McLay, H. A., Needham, E. A. (1984). An exocrine pancreas disease of farmed Atlantic salmon in Scotland. Helgoländer wiss. Meeresunters. 37: 571-586

Poppe, T., Rimstad, E., Hyllseth, B. (1989). Pancreas disease in Atlantic salmon (Salmo salar) postsmolts infected with infectious pancreatic necrosis virus (IPNV). Bull. Eur. Ass. Fish Pathol. 9: 83--85

Poupa, O., Gesser, H., Jonsson, S., Sullivan, L. (1974). Coronary-supplied compact shell of ventricular myocardium in salmonids: growth and enzyme pattern. Comp. Biochem. Physiol. 48A: 85-95

Robinson, W. F., Maxie, M. G. (1985). The cardiovascular system. In: Jubb, K. V. F., Kennedy, P. C., Palmer, N. (eds.) Pathology of domestic animals, Vol. 3, 3rd edn. Academic Press, Orlando, p. $1-81$

Stegeman, J. J., Woodin, B. R., Klotz, A. V., Wolke, R. E., Orme-Johnson, N. R. (1982). Cytochrome P-450 and monooxygenase activity in cardiac microsomes from the fish Stenotomus chrysops. Molec. Pharmac. 21: 517-526

Manuscript first received: December 4, 1989

Revised version accepted: March 6, 1990 